\title{
Art et magie de la dentisterie. Implantologie dans un cas très limite
}

\section{RÉSUMÉ}

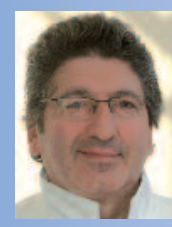

Jean-Michel STROUMZA Ex-assistant hospitalo-universitaire, attaché de consultation à la faculté dentaire de Paris VII, 110 , rue La Boétie, 75008 Paris

France.
Il s'agit d'une réhabilitation occluso-implanto-prothétique à la mandibule d'un édentement ancien d'une trentaine d'années ayant subi une résorption osseuse accélérée par le port de prothèses amovibles unilatérales.

Bien que le pronostic implantaire soit extrêmement réservé sur ce type de crête gingivale en lame de couteau, un traitement implantaire fut proposé en association avec un traitement laser afin d'optimiser les résultats. 
Pourquoi le traitement laser ?

La littérature montre que le laser permet de stimuler tissus durs et tissus mous dans le sens d'une amélioration de la qualité osseuse et gingivale, d'augmenter la respiration cellulaire, la production d'ATP, de fibroblastes, de macrophages, la libération de facteurs de croissance et la néoangiogenèse.

En dermatologie (Dahan, 2004), les coupes histologiques avant et après laser montrent une

\section{Présentation}

Madame L, 60 ans, sans antécédents médicaux, souhaite une solution fixe à un édentement mandibulaire gauche qui I'handicape depuis 30 ans. La gencive est fragile, très fine, en lame de couteau, avec une faible hauteur de gencive attachée (fig. 1).

Le panoramique (fig. 2) montre trois bridges réalisés : 2 au maxillaire et 1 à la mandibule. La motivation principale de réaliser un bridge implanto-porté à droite est de soulager les pressions qui s'exercent sur le bridge gauche en diminuant les surcharges occlusales et de ralentir la formation accélérée de tartre sur le bridge maxillaire droit non fonctionnel.

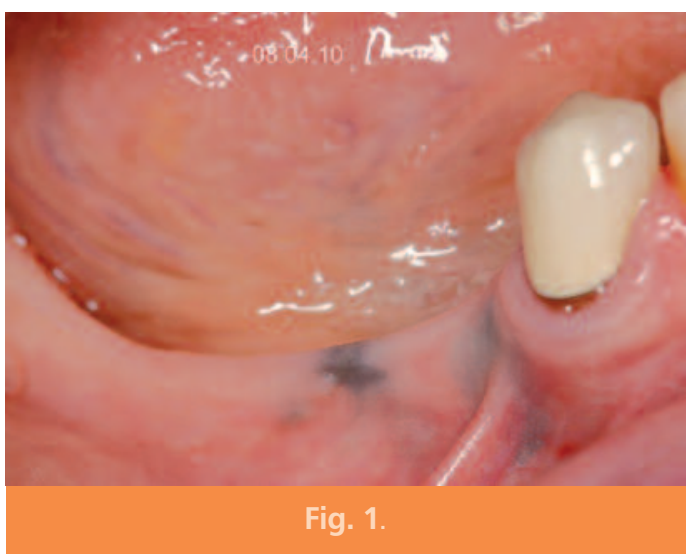

augmentation de l'épaisseur du derme et un rajeunissement des tissus par les apports trophiques grâce à une rapide néoformation de nombreux vaisseaux.

Plusieurs séances de laser seront réalisées avant, pendant et après la chirurgie implantaire : pendant la phase d'ostéo-intégration des implants, pendant et après la mise en fonction des implants et enfin pendant la phase de maintenance.
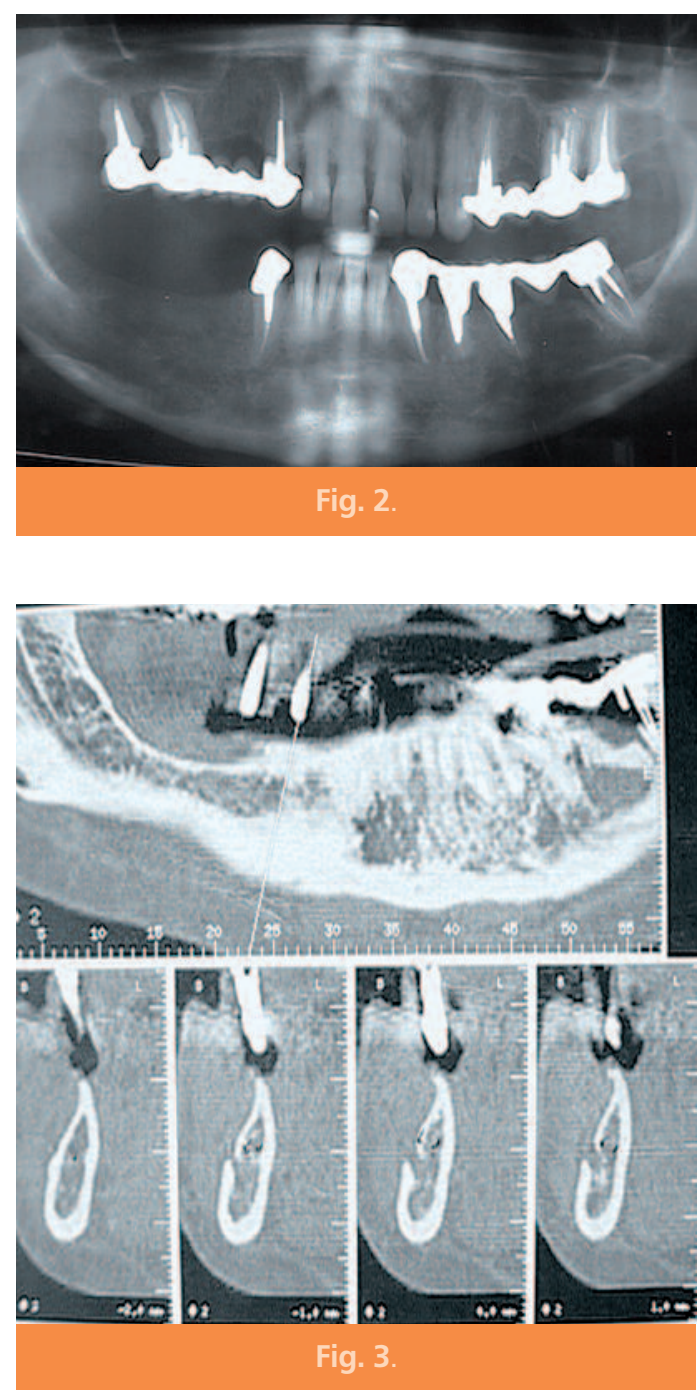
L'analyse scanner (fig. 3) montre une épaisseur osseuse de 1,2 $\mathrm{mm}$ au sommet de la crête, un volume osseux très réduit, un canal mandibulaire à $10 \mathrm{~mm}$ de la crête et un foramen mentonnier très large et très ouvert. Le tissu osseux est de qualité médiocre, niveau 3+ dans la classification de Branemarck.

La superposition d'un transparent (fig. 4) montre la possibilité d'un ancrage primaire sur $3 \mathrm{~mm}$ avec plusieurs spires exposées sur $7 \mathrm{~mm}$.

\section{Thérapeutique}

Après analyse minutieuse de la faisabilité d'implanter trois implants, la décision est prise de préparer préalablement le site de forage. Dans un premier temps, la stimulation gingivale se fait par piquage, scarification et laser associée à un approfondissement vestibulaire (fig. 5).

Cinq semaines plus tard, trois implants sont posés, suivis d'un comblement osseux d'origine bovine recouvert d'une membrane fixée en occlusal par les vis de couverture des implants.

Le site greffé est alors biostimulé au laser sous irrigation abondante d'eau oxygénée 10 volumes.

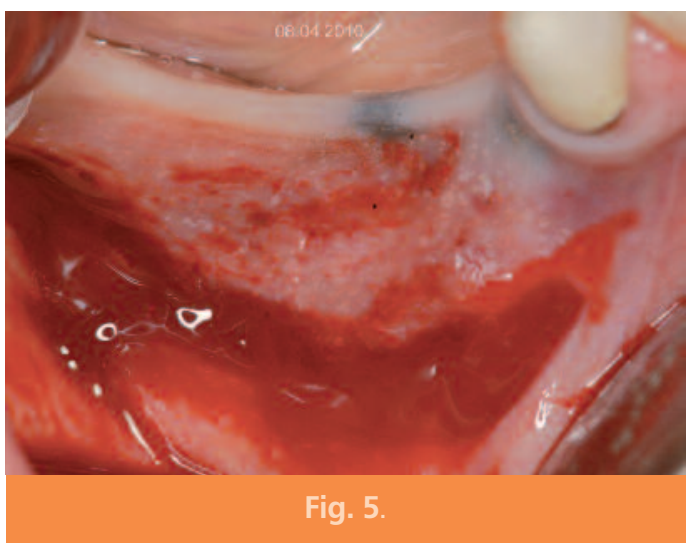

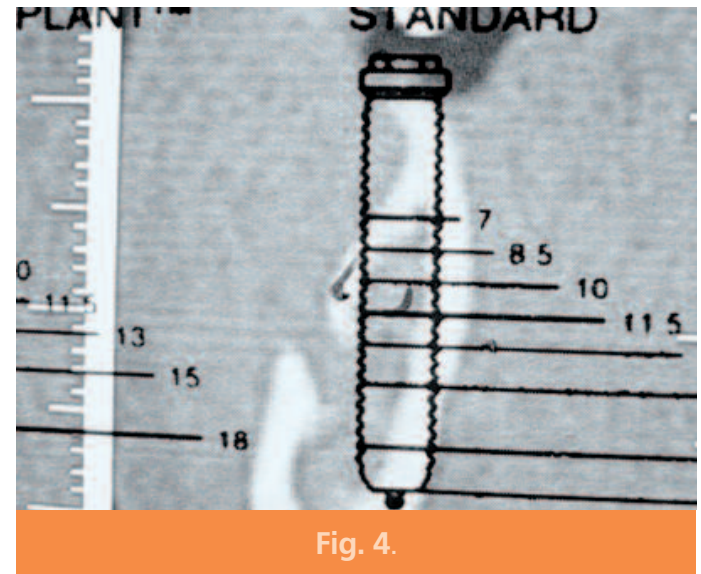

Après anesthésie locale, un lambeau muco-périosté est décollé, mettant à nu un tissu osseux peu épais. En revanche, la gencive réclinée montre une épaisseur intéressante qui a l'avantage de protéger la greffe et dont le saignement signe une vascularisation favorable.

Le forage à $2 \mathrm{~mm}$ de diamètre permet de placer trois tuteurs de parallélisme et de constater que I'os, préalablement préparé selon le principe de stimulation endostée (Bert, 1989), présente un saignement idéal (fig. 6).

Les implants sont ensuite vissés après une préparation adéquate des puits de forage, sous-

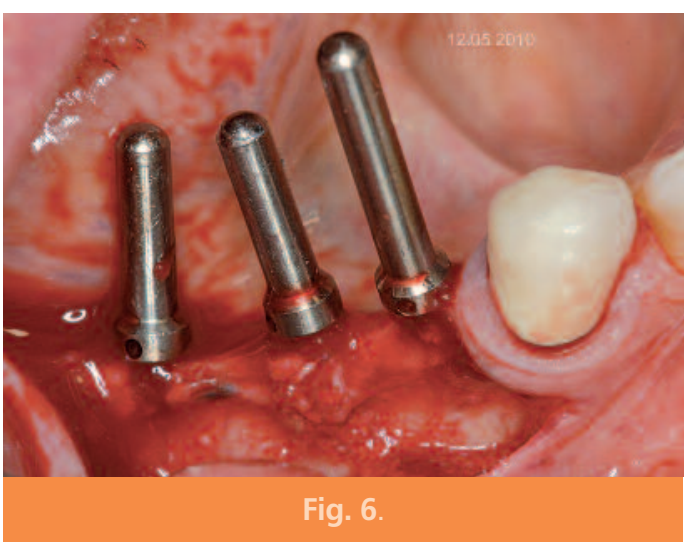


dimensionnés, afin d'obtenir une stabilité primaire recherchée (fig. 7).

Une technique chirurgicale associant vitesse lente de forage et utilisation d'ostéotomes frappés permet d'élargir le diamètre des puits implantaires et d'éviter de trop importantes fenestrations vestibulaires.

La radiographie (fig. 8) montre une hauteur d'os greffé favorable, des implants bien placés. Les vis de couvertures des implants en 44 et 45 sont espacées du col implantaire car elles maintiennent la membrane.

Après la pose des implants, la patiente est hospitalisée pendant une longue période sans lien établi avec l'intervention implantaire. Elle se présente au cabinet 35 jours plus tard, l'ablation des sutures ayant été partiellement réalisée à I'hôpital. Elle présente un déficit d'hy-
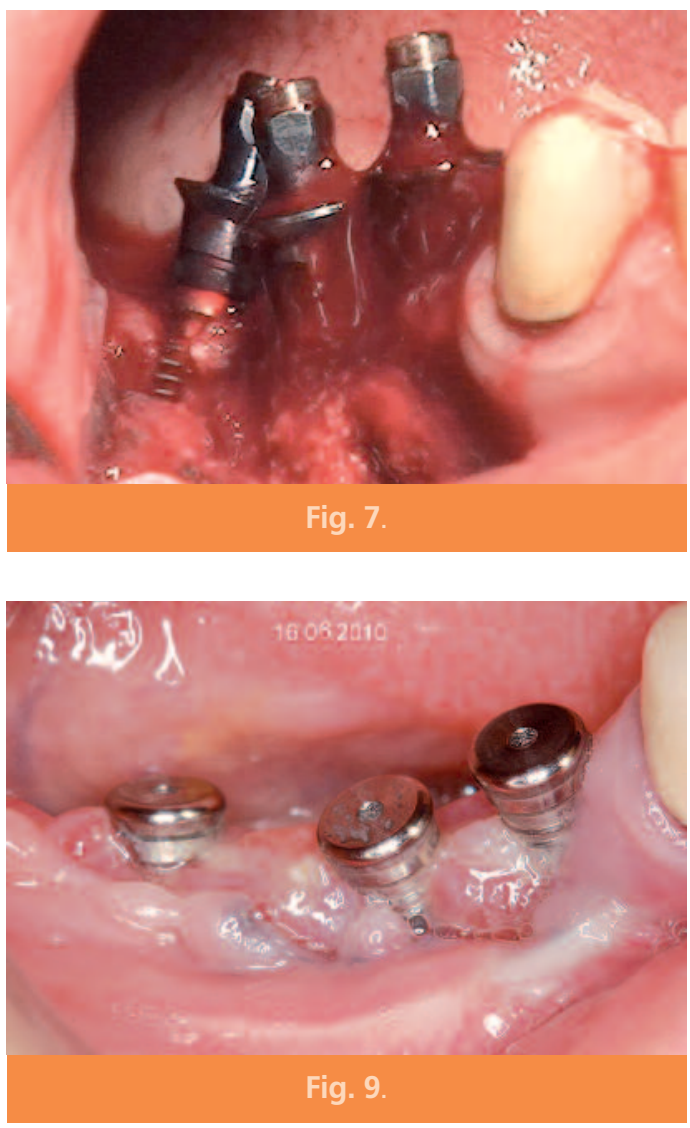

giène conséquent. La greffe osseuse s'est pratiquement totalement résorbée, entraînant la gencive dans sa récession (fig. 9).

Durant les mois suivants, la patiente ne se brosse plus, par crainte d'endommager la zone implantée. À chaque séance, un détartrage approfondi des spires et des zones sous-gingivales est exécuté ainsi qu'une stimulation biologique de la zone implantée au laser (fig. 10). Une nouvelle greffe osseuse recouverte d'une membrane est réalisée six mois plus tard, après surfaçages et traitement laser des spires de titane exposées et décontaminées (fig. 11).

Un réglage du laser réduisant la fluence favorise un bon saignement du site traité. Un réglage augmentant l'effet photothermique en focalisant la fibre optique du laser provoque une coagulation pratiquement immédiate.
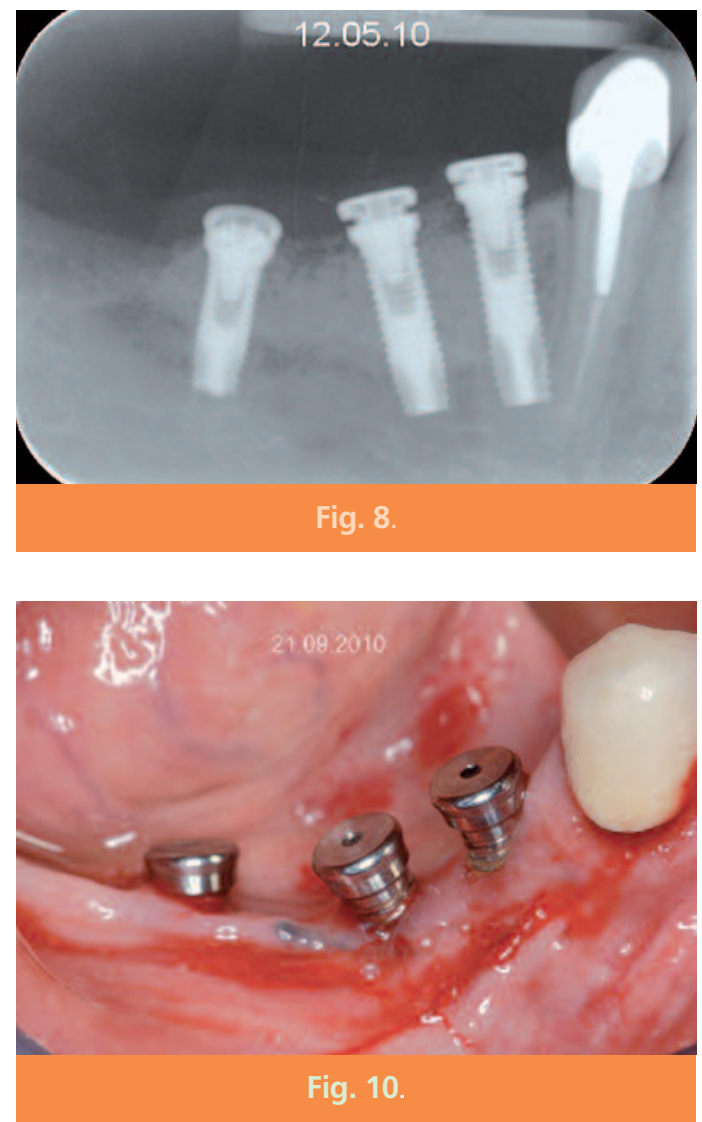

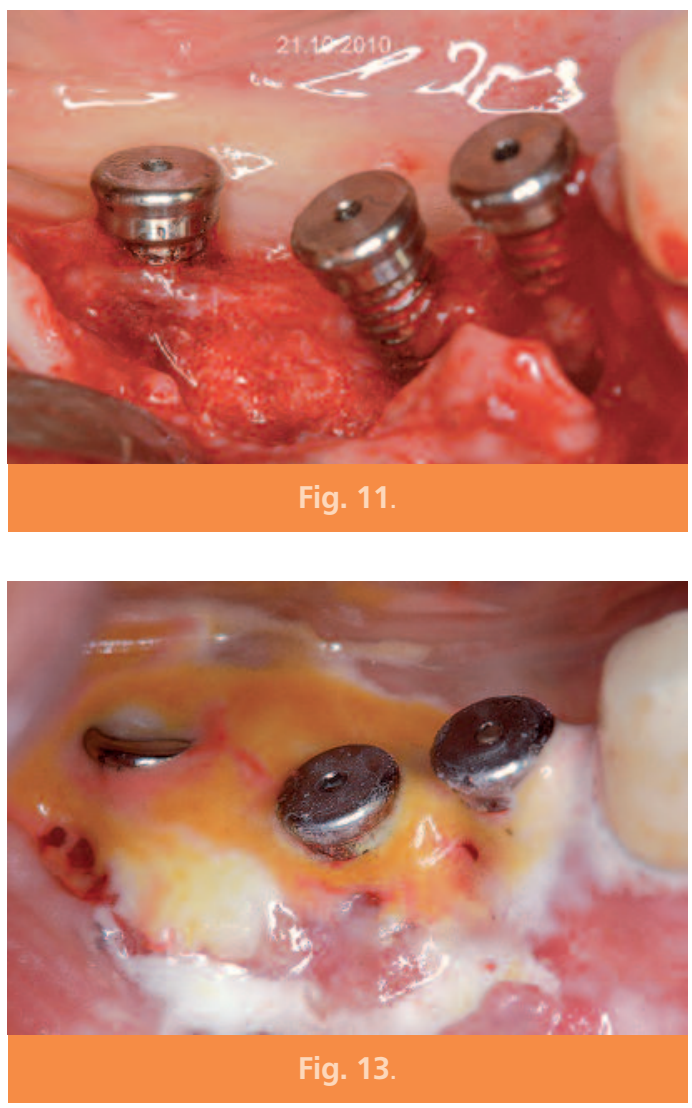

Plusieurs contrôles sont réalisés dans les semaines qui suivent, associant traitements laser et eau oxygénée 10 volumes, parfois en alternance avec de la bétadine.

Les conseils d'hygiène sont rappelés à chaque séance afin de renforcer la motivation du patient.

Enfin, huit mois après la chirurgie implantaire, la patiente présente une qualité d'hygiène irréprochable.

La mise en fonction avec mise en charge provisoire simultanée est réalisée après un hydropolissage puissant et une irradiation laser sous polyvidone iodée (fig. 13).

Les faux moignons sont vissés avec un torque de $32 \mathrm{Ncm}$ et préparés de dépouille ${ }^{(*)}$ dans la
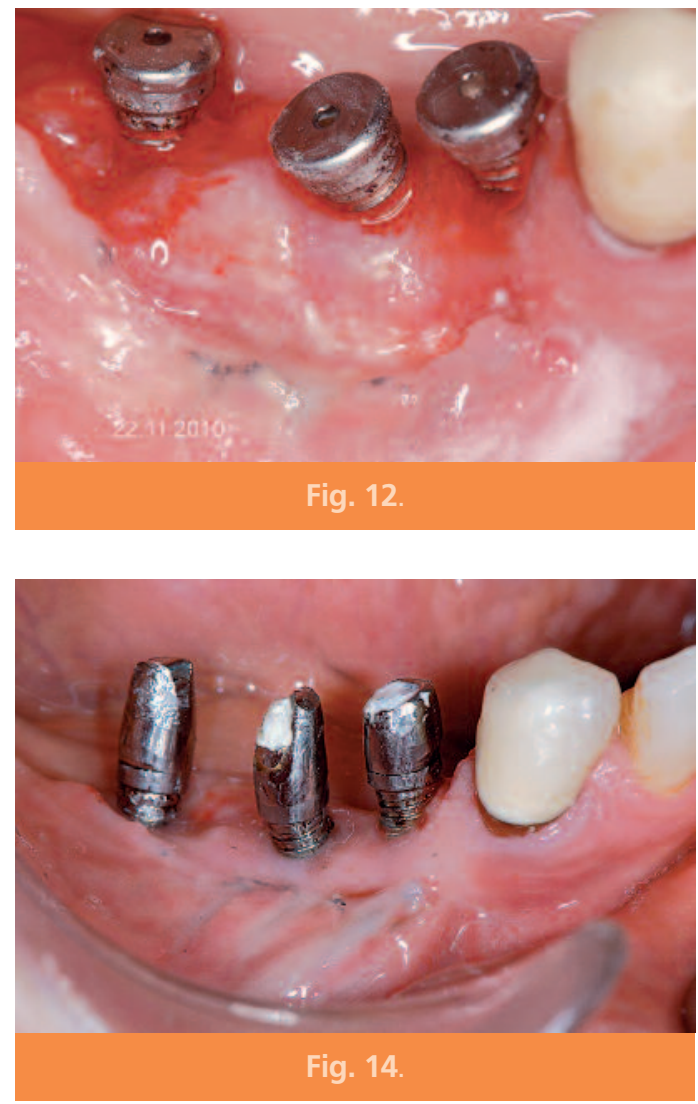

mesure du possible. La déhiscence gingivale qui accompagne la résorption des comblements osseux et l'exposition non désirée des spires implantaires amène à envisager un type de réalisation prothétique d'un genre particulier et totalement inhabituel (fig. 14).

La radiographie rétroalvéolaire met en évidence les zones de contre-dépouille cervicales impossible à corriger sans risquer de léser la vis de transfixation de l'inlay core sur l'implant (fig. 15).

Le bridge provisoire réalisé est une solidarisation à la résine des trois couronnes ajustées séparément sur chaque élément (fig. 16).

Le travail de prothèse de J.F. Mariotti est constitué d'un bridge de trois éléments céramo-

$\left.{ }^{*}\right)$ C'est-à-dire permettant l'insertion et la désinsertion aisée d'une prothèse fixe. Les inlay core sont préparés de forme tronconique avec des génératrices convergentes vers la zone occlusale. 

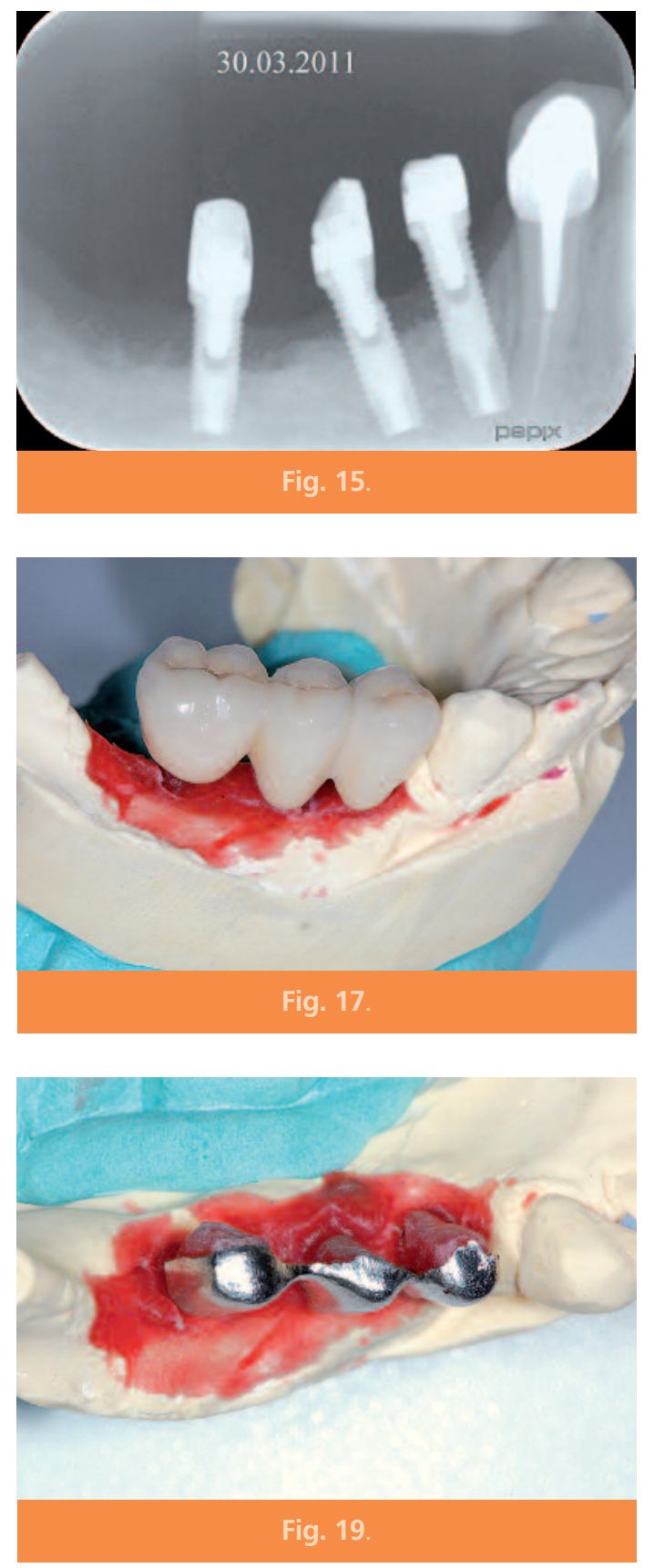

métalliques qui s'articule sur deux hémi-structures métalliques qui se placent latéralement en vestibulaire et en lingual, en enserrant les trois inlay cores qui seront emprisonnés par la supra structure céramo-métallique (fig. 17 et 18). La vue occlusale montre la demi-armature vestibulaire mise en place latéralement (fig. 19).
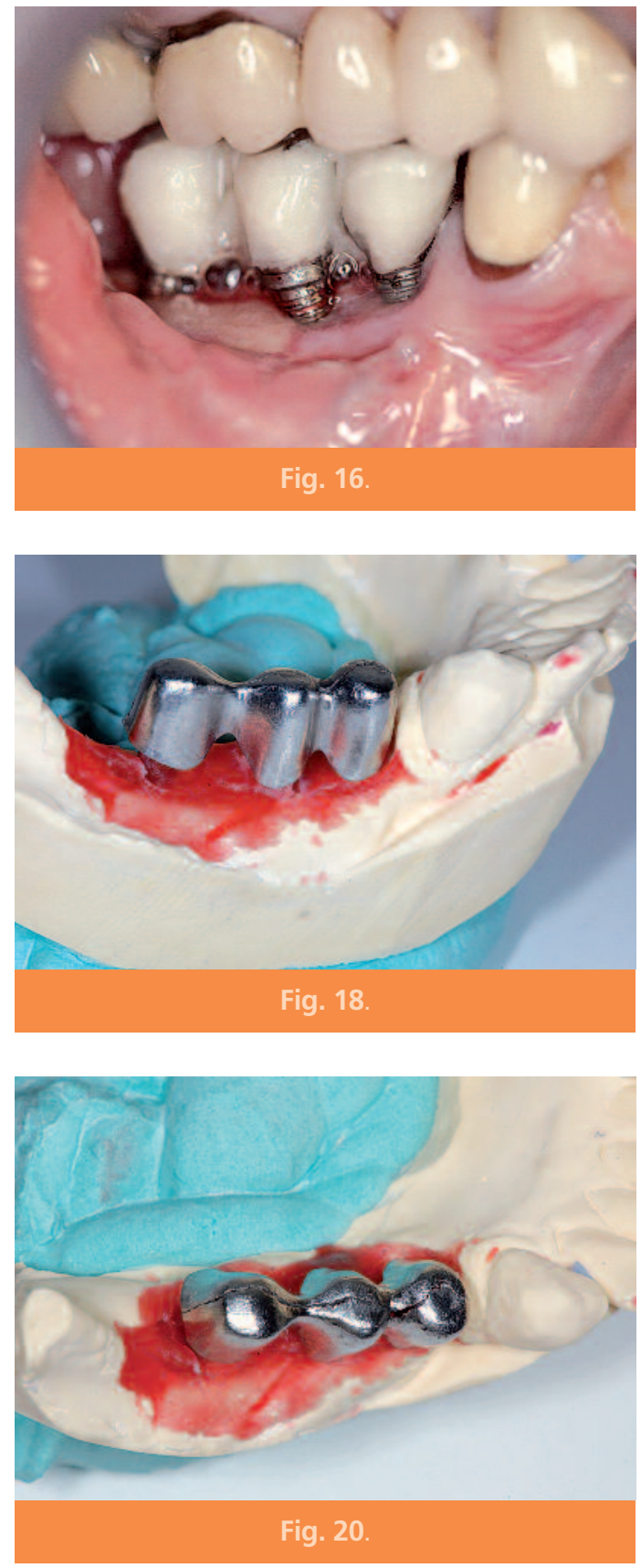

Les trois photographies suivantes représentent la partie technique de cet assemblage prothétique en trois parties pour s'affranchir du manque de parallélisme des trois faux moignons et des contre-dépouilles s'étendant du col implantaire à la limite osseuse cervicale. 


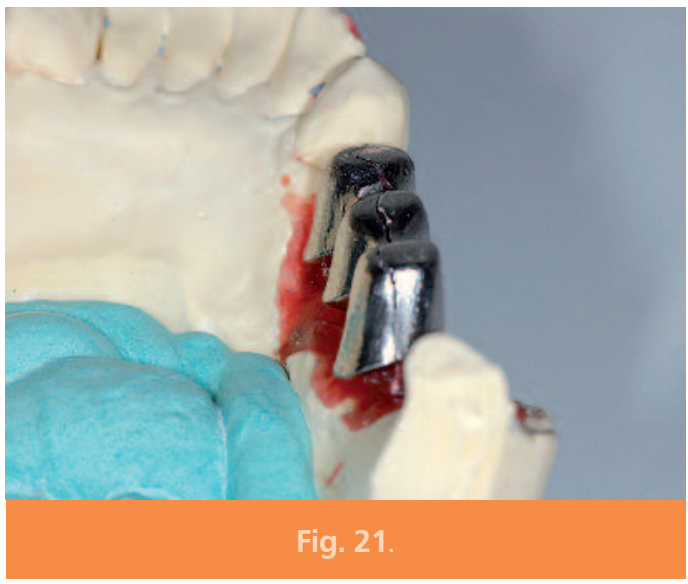

La vue occlusale sur les modèles en plâtre montre la ligne de jonction des deux hémistructures métalliques (fig. 20).

La vue distale montre la zone d'articulation des deux éléments (fig. 21).

La vue linguale montre le volet métallique lingual qui, en se plaçant sur la partie linguale des

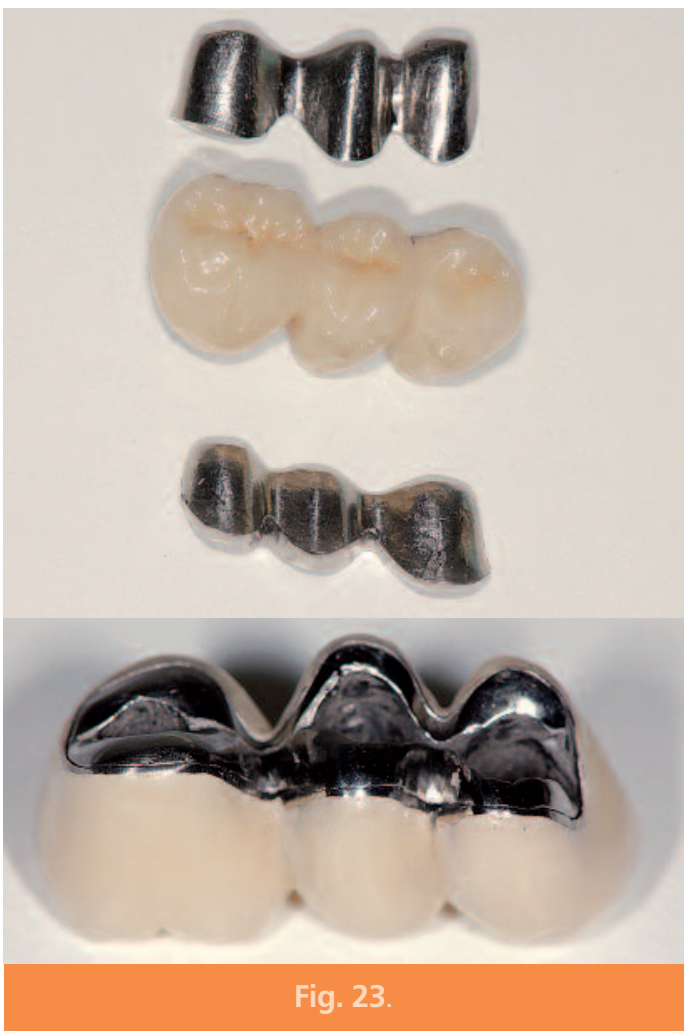

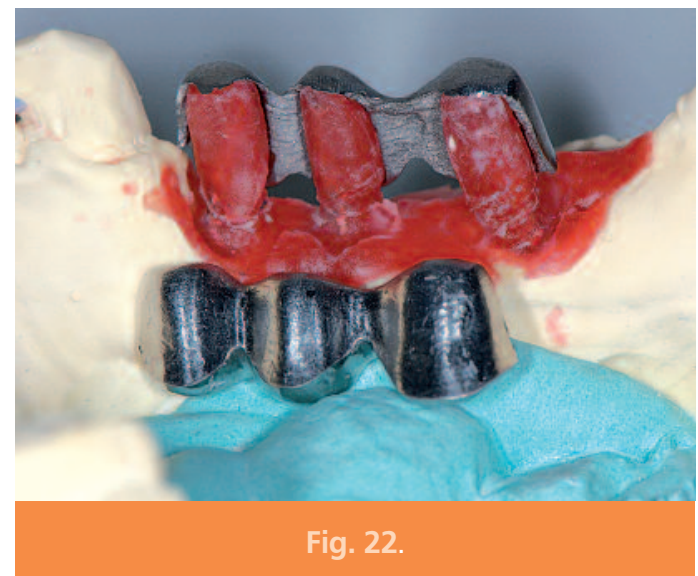

faux moignons, va s'articuler avec le volet vestibulaire déjà en place (fig. 22).

La photographie suivante (fig. 23) montre la réalisation prothétique éclatée et les trois parties du bridge qui seront solidarisées et scellées définitivement ensemble de façon à assurer un système rigide, indéformable et impossible à desceller.

L'état de la gencive s'est amélioré après le coatching lors des multiples contrôles.

Les nombreuses interventions de décontaminations, d'assainissements et de stimulations de tous les éléments biologiques tissulaires et immunitaires au laser ont favorisé cette cicatrisation (fig. 24).

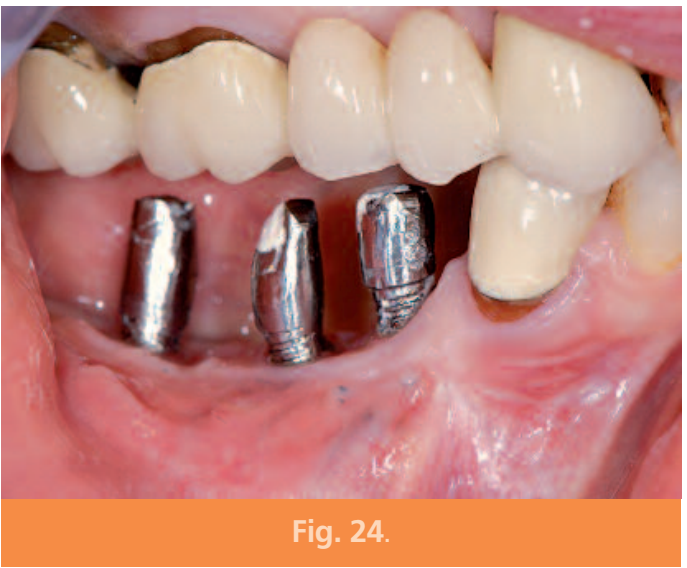


La radiographie (fig. 25) montre le maximum de dépouille obtenu par fraisage in situ sans fragiliser les inlay cores.

Le manque de dépouille dans l'axe des faux moignons a été compensé par un système astucieux en utilisant la dépouille dans un axe perpendiculaire à l'axe implantaire c'est-à-dire les zones latérales vestibulaire et linguale : les deux
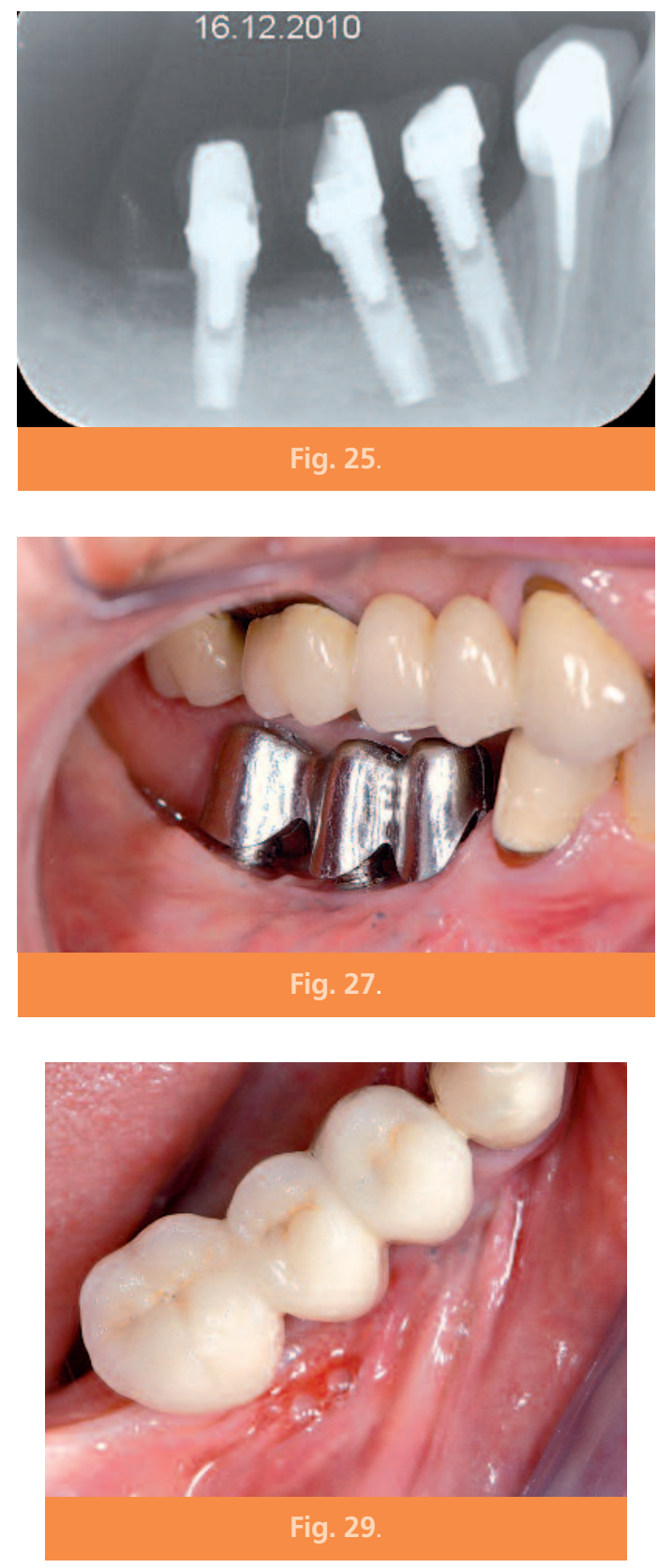

parties métalliques s'unissent comme deux hémi-télescopes et sont scellées dans cette position et fermement maintenues par le scellement extemporané de la superstructure céramo-métallique.

La vue clinique vestibulaire montre le contact intime du volet métallique lingual qui s'agrège sur les trois inlay cores (fig. 26).
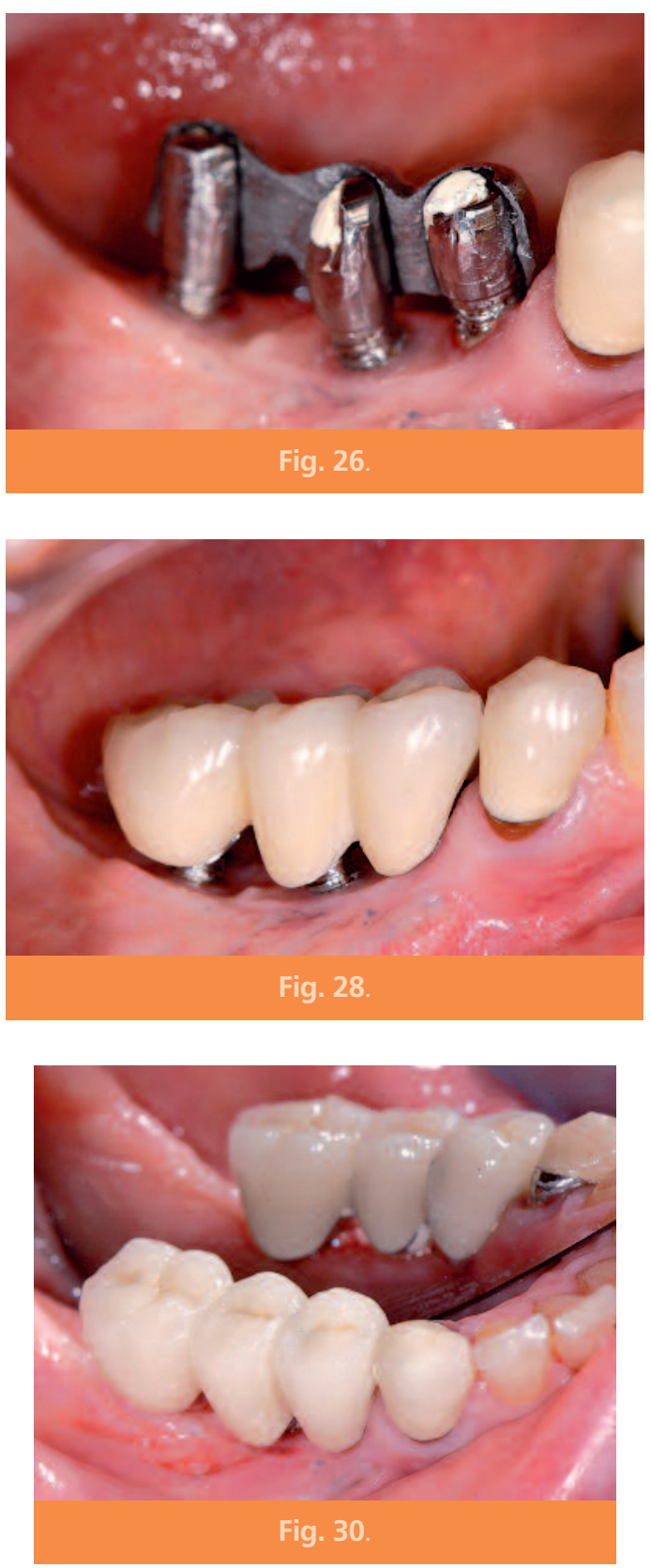
Le volet vestibulaire emboîte la partie vestibulaire des trois inlay cores (fig. 27).

Le bridge en place maintient fermement les deux hémi-téléscopes (fig. 28).

La vue du bridge céramo-métallique montre une bonne intégration occlusale (fig. 29).

Vue vestibulaire et linguale par réflexion dans le miroir du bridge après scellement (fig. 30). La vue clinique à un an postimplantaire montre une intégration gingivale satisfaisante, juste récompense après les étapes difficiles franchies.

Le contrôle radiographique (fig. 31) objective un état osseux convenable.

Les figures 32 et 33 montrent à 19 mois une stabilité clinique et radiographique.
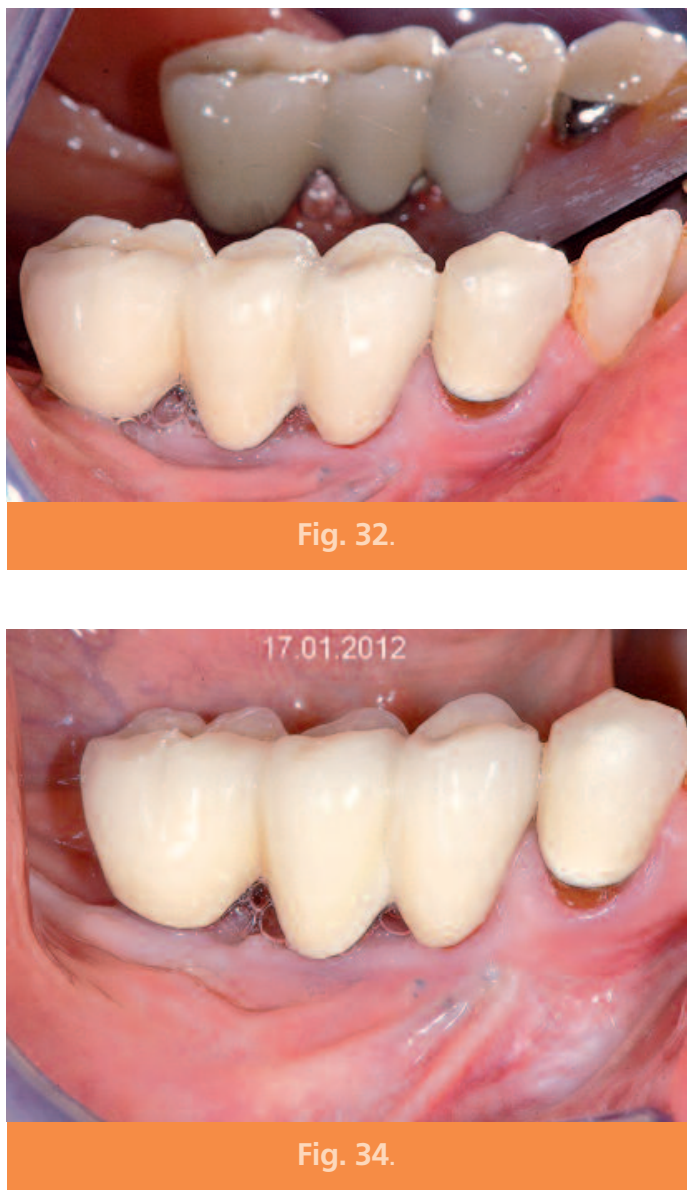

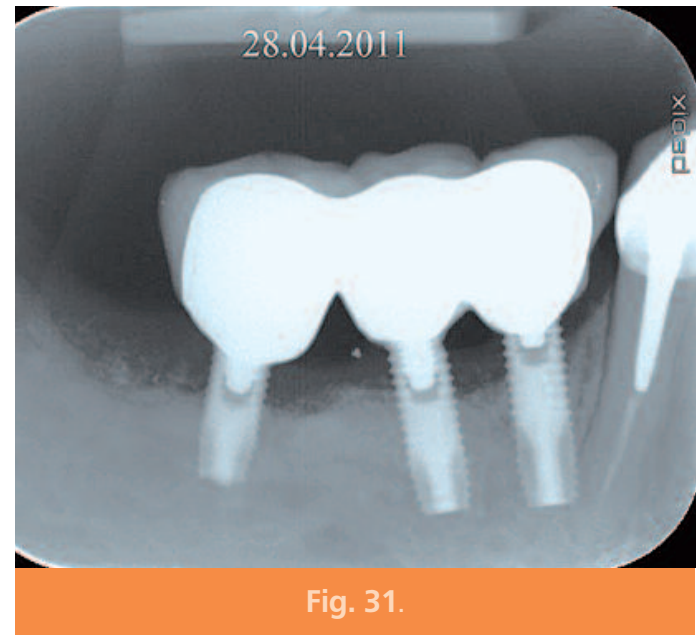

À 21 mois, la patiente, à l'écoute de nos conseils d'entretien et d'hygiène, montre une bonne intégration gingivale (fig. 34 et 35 ).
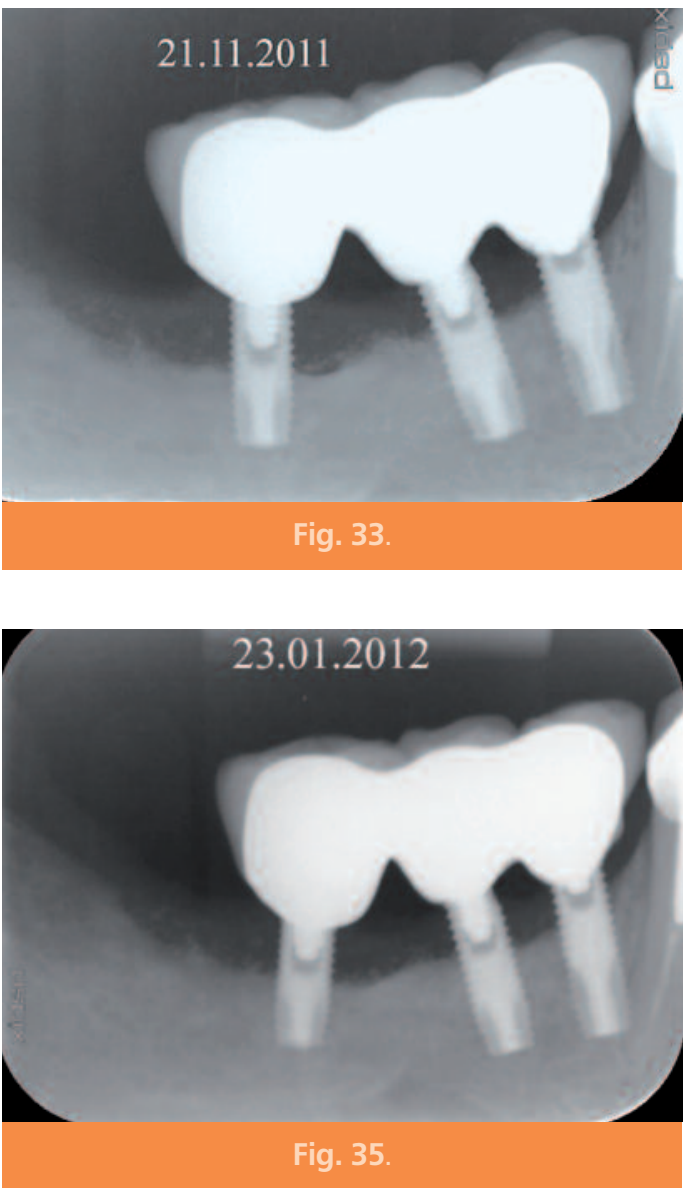
Malgré les nombreuses phases de découragement de la patiente objectivées par une absence totale d'hygiène qui aurait pu mettre en péril le projet de prothèse implanto-portée, la situation s'est nettement améliorée quand la patiente a collaboré. Cette implication est fondamentale pour mettre en place tous les éléments fondateurs de la pérennité de ce travail particulièrement délicat.

\section{Ouvrages à consulter}

- Atwood DA.

Reduction of residual ridges:

a major oral disease entity.

J Prosth Dent

1971; 26:266-79.

- Bert M.

La stimulation endostée

en implantologie ; études

et résultats après 2 ans.

Cah Prothèse 1989;65:23-30.

- Blum JY, Michailesco P,

Abadie MJ.

An evaluation of

the bactericidal effect of

the Nd:Yap Laser.

J Endod 1997;23:583-5.

- Branemark PI, Zarb GA,

Albrektsson T.

Prothèses ostéo-intégrées.

L'ostéo-intégration

en pratique clinique.

Paris : CDP, 1987.

- Caccianiga GL, Papagna R, Baldini A.

Analyse de la littérature

et évaluation in vivo

des effets

de l'application du laser

dans les soins conservateurs.

Doctor OS 2002; mars 2002.

- Crespi R, Covani U,

Margarone JE, Andreana S.

Periodontal tissue regeneration on beagle dog laser therapy.

Lasers Surg Med

1997;21:395-402.

- Dahan S, Michaud T.

Les lasers en dermatologie.

Rueil-Malmaison : Doin, 2004.
- Darbar R. Hopper C, Speight PM, Newmann HN. Combined treatment approach to gingival overgrowth due to drug therapy. J Clin Periodontol 1996;23:941-4.

- Kesler G, Shvero DK, Tov YS, Romanos G.

Platelet derived growth factor secretion and bone healing after Er:YAG laser bone irradiation.

J Oral Implantol 2011;37 Spec No:195-204.

- Kiernicka M, Owczarek B, Galkowska E, Wysokinska-Miszczuk J. Comparison of the effectiveness of the conservative treatment of the periodontalpockets with or without the use of laser biostimulation. Ann Univ Mariae Curie Sklodowska [Med]. 2004;59(1):488-94.

- Missika P, Stroumza JM. Traitement de péri-implantite avec un laser $\mathrm{Nd}:$ YAP. ROS 2003;32:215-29.

- Missika P, Rey G, Stroumza JM. Les lasers et la chirurgie dentaire.

Chapitre 3 : place réelle du laser en omnipratique quotidienne. Chapitre 6 : Intérêt du laser dans le traitement de la péri-implantite.
Rueil-Malmaison : CdP,

Coll. JPIO, 2009.

- Pietrokowski J, Massler M. Alveolar ridge resorption following tooth extraction. J Prosthet Dent 1967;17:21-7.

- Ricardo A, et al. In vivo study of the $\mathrm{Nd}$ : YAP laser in persistent periapical lesion. Photomed Laser Surg 2005;23:582-5.

- Rocca JP. Les lasers en odontologie. Paris : CDP, Coll. Memento, 2008.

- Sauvetre E, Rastegar B, El Yazami H.

Evaluation Clinique du laser YAP en parodontologie.

Inf Dent 2004;16(23 avr.):1077-82.

- Small PN, Tarnow DP. Gingival recession around implants: a 1-year prospective study. Int J Oral Maxillofac Implants 2000;15(4):527-32

- Sulc J, Jelinkova $H$, Jabczynski JK, Zendzianb W, Kwiatkowski J, Nejezchleb K, Skoda V.

Comparison of diode-side-pumped $\mathrm{Nd}$ : YAG and Nd: YAP laser - Solid State Lasers XIV: Technology and Devices.

Edited by Hoffman HJ, Shori RK, Ramesh K. 
Proceedings of the SPIE 2005;5707:325-34.

- Stroumza JM. Option implantaire dans un cas limite. Inf Dent 2006;88(3)(18 janv.):74-9.

- Stroumza JM.

Traitements implantaires simplification d'un cas complexe. Act Odontol Stomatol 2003 (sept);223:223-30.

- Stroumza JM.

Prévention et gestion des complications de greffes osseuses. Act Odontol SStomatol 2006;235:287-94.

- Stroumza JM. Implantologie sans greffe. In : Les 10 points clés en implantologie. Européenne Edition, Coll. Les 10 points clés en odontologie, nov. 2009.

- Stroumza JM. Laser Nd YAP et réimplantation. Inf Dent 2010;5(3 févr.).
- Stroumza JM.

Biostimulation au laser et implantologie.

Alpha Oméga News 2010 (avril);132:14-8.

- Trisi P, Lazzara R, Rao W, Rebaudi W. Bone-implant contact and bone quality: evaluation of expected and actual bone contact on machined and osseotite implant surfaces. Int J Periodontics Restorative Dent 2002;22(6):535-45.

\section{Questions-Réponses}

\section{>1. - Qu'est-ce-que la fluence d'un laser?}

La fluence est un concept utilisé pour décrire l'effet du rayonnement laser sur la matière dans le temps.

C'est la puissance en watts (W) multipliée par le temps d'exposition et divisé par la surface de l'impact lumineux en $\mathrm{cm}^{2}$.

Fluence $\left(\right.$ joules $\left./ \mathrm{cm}^{2}\right)=\frac{P(w) \times \operatorname{tps}(\mathrm{s})}{\text { Surface }\left(\mathrm{cm}^{2}\right)}$

Une surface d'impact importante signifie que l'énergie du laser est distribuée sur une plus grande étendue et donc que son intensité est plus faible, tandis qu'une surface plus petite permet de concentrer l'énergie laser

En conséquence la biostimulation sera obtenue avec des intensités plus faibles et surfaces plus grandes donc avec une fluence plus petite de l'ordre de 0,05 joules $/ \mathrm{cm}^{2}$ à 0,5 joules $/ \mathrm{cm}^{2}$ et un temps d'exposition de 30 à 120 secondes.

\section{2. - Qu'apporte le protocole laser + eau oxygénée 10 volumes ?}

Ce protocole associe le traitement classique et l'action bactéricide du laser sous irrigation abondante d'eau oxygénée.

On obtient ainsi un assainissement complet de la zone découverte de l'implant et une décontamination très efficace de la surface de l'implant, surtout si cette surface est rugueuse, ce qui est le cas de la majorité des implants actuels. Le laser utilisé pour l'intervention est le laser $\mathrm{Nd}-\mathrm{YAP}$, fabriqué par Lokki ${ }^{\circledR}$, pulsé, de fréquence $1,34 \mu$ avec une fibre optique de $320 \mu$ de diamètre. En position de travail " gencive moins ", c'est-à-dire à $30 \mathrm{~Hz}$ de fréquence et $170 \mathrm{~mJ}$ d'énergie par impulsion, il développe une puissance moyenne de $5 \mathrm{~W}$ et donc a une puissance de crête de 1,1 KW.

Après exérèse totale du tissu de granulation, toute cette zone est abondamment irriguée 
avec de l'eau oxygénée à 10 volumes. Les irrigations sont immédiatement suivies de tirs lasers en rafales de quelques secondes, espacés de brefs temps d'arrêt.

Le geste doit être souple, précis, en balayage parallèle aux parois de l'implant. Le praticien doit soigneusement éviter de diriger le faisceau laser sur le titane, ce qui risquerait de créer des impacts. Le faisceau doit balayer le fond de l'interface os implant parallèlement à celui-ci.

\section{Remarque}

L'association du laser avec l'eau oxygénée à 10 volumes confère une action bactéricide efficace sur la surface contaminée de l'implant, sur les tissus durs et sur les tissus mous.

Cette opération est renouvelée plusieurs fois, entrecoupée de brèves séquences de repos.

L'effet bactéricide du laser Nd YAP est potentialisé lors de son association avec une irrigation d'eau oxygénée à $3 \%$ ou 10 volumes.
Cette potentialisation est sans doute due à la transformation in situ des molécules de $\mathrm{H}_{2} \mathrm{O}_{2}$ sous l'action thermique du laser en ion $\mathrm{OH}-$-, $\mathrm{O}_{2}$ - et molécules $\mathrm{H}_{2} \mathrm{O}$. Par ailleurs, il semble possible qu'une très faible quantité d'ozone $\left(\mathrm{O}_{3}\right)$ soit aussi libérée. Les ions $\mathrm{O}_{2}$ - et $\mathrm{OH}-$ libérés auront une action bactéricide sur la flore sous-gingivale environnante, majoritairement flore anaérobie stricte. La faible quantité d'ozone libéré in situ pourrait elle aussi intervenir par stimulation locale du système immunitaire.

Enfin, les dernières recherches montrent une formation d'oxygène singulet (forme non naturelle dudit oxygène) lorsque les photons viennent impacter l'oxygène de l'eau oxygénée.

Cette molécule riche en énergie s'isomérise en émettant une lumière rouge avec une demi-vie de 0,1 seconde en oxygène triplet $\left(\mathrm{O}_{2}\right.$ paramagnétique) la forme naturelle dudit oxygène.

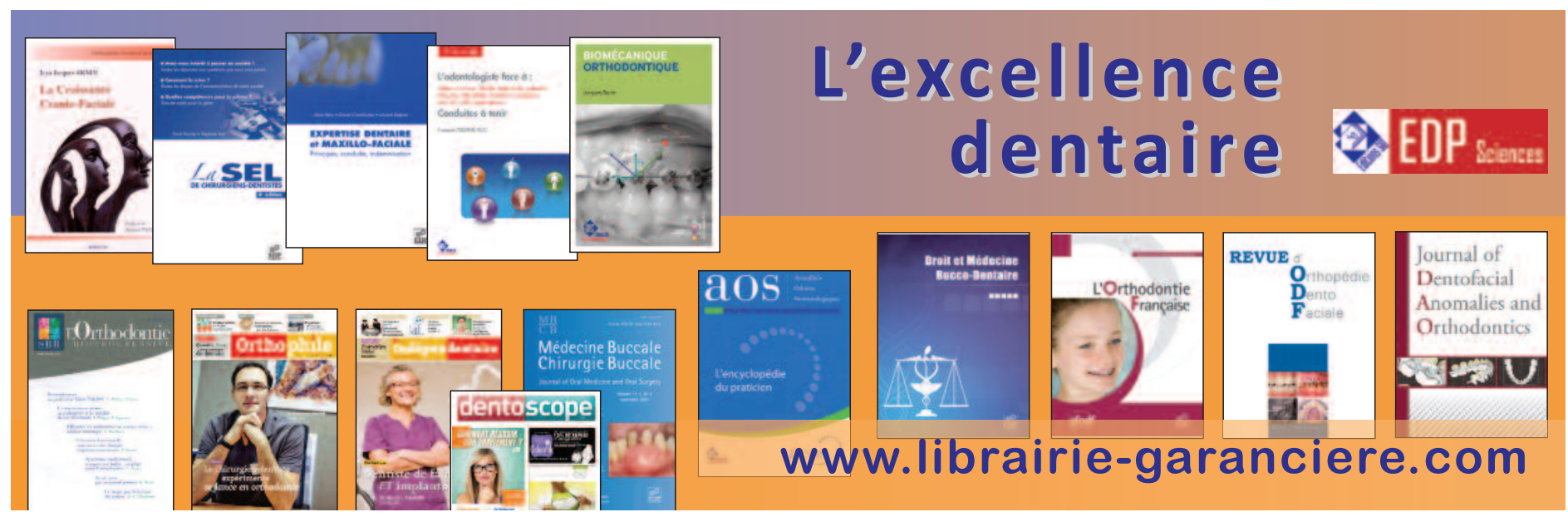

